\title{
BMJ Open Establishing best practices in cancer online support groups: protocol for a realist review
}

\author{
Jacqueline L Bender (D) , ${ }^{1,2}$ Stephanie Babinski, ${ }^{1}$ Geoff Wong (D) ${ }^{3}$ \\ Andrea C Tricco (D) , ${ }^{4}$ Marina Englesakis (D) , ${ }^{5}$ Alaina B Cyr, ${ }^{6}$ Henry Potts (D) , ${ }^{7}$ \\ Olga Perski (D) , Mary Jane Esplen, ${ }^{8}$ Colleen Young (D) , ${ }^{10}$ Richard Wassersug (D) ,11 \\ Victoria Forster (D) , ${ }^{12}$ Janet Papadakos (D) , ${ }^{13}$ Charlene Soobiah (D) , ${ }^{14}$ \\ Colleen Fox, ${ }^{15}$ Alison Gothard-Huang, ${ }^{1}$ Holly Witteman (D) ${ }^{16}$
}

To cite: Bender JL, Babinski S, Wong G, et al. Establishing best practices in cancer online support groups: protocol for a realist review. BMJ Open 2021;11:e053916. doi:10.1136/ bmjopen-2021-053916

- Prepublication history for this paper is available online. To view these files, please visit the journal online (http://dx.doi. org/10.1136/bmjopen-2021053916).

Received 27 May 2021 Accepted 24 September 2021

Check for updates

(c) Author(s) (or their employer(s)) 2021. Re-use permitted under CC BY-NC. No commercial re-use. See rights and permissions. Published by BMJ.

For numbered affiliations see end of article.

Correspondence to Dr Jacqueline L Bender; jackie.bender@uhnresearch.ca

\section{ABSTRACT}

Introduction Considerable observational evidence suggests that cancer online support groups reduce feelings of isolation, depression and anxiety, enhance coping and self-management, and lead to better informed patients. Other studies indicate that cancer online support groups can increase distress. Yet no studies theorise the complex, context-dependent mechanisms by which cancer online support groups generate their-sometimes contrasting-outcomes.

Methods and analysis Guided by an integrated knowledge translation approach and the strategy for patient-oriented research, we will conduct a realist review of cancer online support groups in partnership with stakeholders. We will follow Pawson's five steps and existing quality standards to develop a program theory that explains how cancer online support groups work, for whom and in what circumstances. The specific research questions will be: what positive and negative outcomes have been reported on cancer online support groups? What are the mechanisms that are associated with these outcomes, in which contexts and for whom? Through a rigorous review of relevant scientific and grey literature, as well as ongoing dialogue with stakeholders, a program theory will be developed to explain who benefits from cancer online support groups and who does not, what benefits they derive (or do not), and the factors that affect these outcomes.

Ethics and dissemination The use of secondary data for this review precludes the need for ethical approval. Dissemination will be informed by the knowledge-toaction framework and will consist of tailored knowledge products that are conceived of collaboratively with stakeholders. These will include peer-reviewed publications on how cancer online support groups can be optimised and best practice recommendations to maximise the benefits experienced by people with cancer. These traditional scientific outputs, along with their respective evidence summaries, will be amplified through strategic social media events hosted and promoted by knowledge users.

PROSPERO registration number CRD42021250046.

\section{Strengths and limitations of this study}

- This will be the first realist review to explore how cancer online support groups work, in what circumstances, for whom and why.

- The realist approach will help to uncover the context-dependent mechanisms by which cancer online support groups generate their outcomes.

- Involvement of stakeholders and patient partners will ensure relevance of the program theory and knowledge products.

- The predominance of literature on breast cancer online support groups may limit the generalisability of findings.

\section{INTRODUCTION}

Cancer is the leading cause of death, premature death and years of life lost worldwide. ${ }^{1}$ In 2020, 19.3 million people were diagnosed with cancer, and 9.6 million died from it. ${ }^{2}$ The number of new cases of cancer is expected to increase to 30.2 million by $2040 .^{2}$ With advances in detection and treatment, it is estimated that over two-thirds of people who are diagnosed with cancer will become long-term survivors. $^{2}$

Although survival rates continue to improve, the diagnosis and treatment of cancer is associated with multiple physical and psychosocial challenges that can reduce overall quality of life. ${ }^{34}$ Yet, many patients and survivors struggle with the effects of cancer and its treatment without much professional help or support. ${ }^{56}$ As a result, many cancer patients and survivors report being dissatisfied with the information they receive from healthcare professionals ${ }^{7}$ and experience high levels of unmet supportive care needs. ${ }^{89}$ Addressing the supportive care needs of the growing population of cancer patients and 
survivors will require innovative strategies and delivery methods. ${ }^{56}$

Cancer patients and survivors may be able to address many of their supportive care needs with support from peers. ${ }^{10}{ }^{11}$ Peer support is an important complement to the supportive care provided by health professionals and is highly valued by patients with cancer, ${ }^{12}{ }^{13}$ even for those with support from family and friends. ${ }^{14}$ Support from other patients with cancer and survivors provides reassurance, practical know-how and emotional support that cannot be gained from other supportive relationships. ${ }^{15}$ Further, research has shown that social support has a direct effect on physical and mental health, and may extend cancer survival. ${ }^{16}$

Online support groups offer a promising means of reaching a wider segment of cancer patients and survivors at a reduced cost. ${ }^{10}$ Online support groups, also known as online communities, are virtual social spaces where people with a common condition or shared interests come together to get and give information and support, to learn or to find company. ${ }^{17}$ They may be independent discussion forums or embedded within popular social media platforms, like Facebook and Twitter. Some of them are moderated by community managers or health professionals; others are not. Research suggests that as many as one in four cancer patients and survivors will turn to online support groups to seek information and support from peers. ${ }^{11}$

Considerable observational evidence suggests that online support groups reduce feelings of isolation, depression and anxiety, and enhance knowledge, coping and self-management among cancer patients and survivors. ${ }^{18-24}$ Studies also suggest that adding an online support group to a digital health intervention can reduce attrition, enhance engagement and increase effect sizes. ${ }^{25}{ }^{26}$ However, online support groups are not riskfree. A randomized controlled trial (RCT) comparing an unmoderated online support group for newly diagnosed patients with breast cancer reported an increase in distress among participants in the intervention group. ${ }^{27}$ Paradoxically, $60 \%$ of participants reported high levels of satisfaction with the online support group and some took the initiative to establish an online support group of their own after the study had ended.

Overall, there is a lack of clear evidence on the effects of cancer online support groups, along with how and why they work or do not work. Prior systematic reviews of cancer online support groups have found a heterogeneous evidence base and few have examined their underlying mechanisms. ${ }^{28-31}$ A 2017 systematic review of six RCTs of breast cancer online support groups found a small to moderate reduction in depression among online support group users compared with controls, and no significant improvements in anxiety or quality of life. ${ }^{31}$ No differences in outcomes in online support groups led by peers compared with those led by health professionals were found. However, the qualitative data collected in many of the trials highlighted benefits experienced by the participants that were not captured by the self-report measures. These findings suggest that online support groups are complex, what works for one person or in one setting may not work for others or in other settings. Further, they indicate a need to explore the choice of outcomes measured.

In sum, there is a clear need, which has been confirmed by the knowledge users and patient partners on the project team, for a better understanding of how and why cancer online support groups work in order to optimise their effects. A realist review can address these questions and has been successfully used to help explain how other complex healthcare interventions can be optimised. ${ }^{32-35}$ However, no realist reviews on the subject have been found. Our project intends to address this knowledge-topractice gap.

\section{Study design}

We will conduct a realist review to investigate how and why cancer online support groups work, in what circumstances and for whom. A realist review is a theory-driven systematic review of the literature that is useful for making sense of diverse outcome patterns from complex, underconceptualised topics with a heterogeneous evidence base. ${ }^{36}$ Traditional systematic reviews are limited in their ability to unpack conflicting evidence from heterogeneous studies, and cannot always explain which intervention components are responsible for which effects. ${ }^{37}$ Realist reviews aim to build and test theories that explain how complex interventions work by examining the relationships between the contexts in which they operate, the mechanisms by which they work, and the different outcomes they produce. ${ }^{36}$

The realist philosophy of science offers a useful way of understanding causation. ${ }^{38}$ Realism assumes that nothing works in every setting or for everyone, and that outcomes are context-dependent. The outcomes in an intervention happen (or not) because the participants react or make particular decisions in response (or not) to contextual factors. Things that may function as context can include participant characteristics, values, beliefs, norms, cultural roles and previous experiences. ${ }^{38}$ Hence, many realist reviews commonly consider the underlying psychological or behavioural processes that interact with contextual factors to produce particular outcomes. These underlying causal processes, known as mechanisms, are sensitive to variations in context; they fire when the contexts are right. Thus, the causal explanations produced in realist reviews take the form of 'Context-Mechanism-Outcome Configurations', or CMOCs, as they are commonly known. CMOCs explain what is functioning as a context, to trigger what mechanism, resulting in what outcome. ${ }^{38}$ In the case of cancer online support groups, a realist review can help to uncover the mechanisms that produce different outcomes for different populations of people with cancer in different circumstances.

The realist review methodology begins and ends with theory. It involves developing an initial program theory 


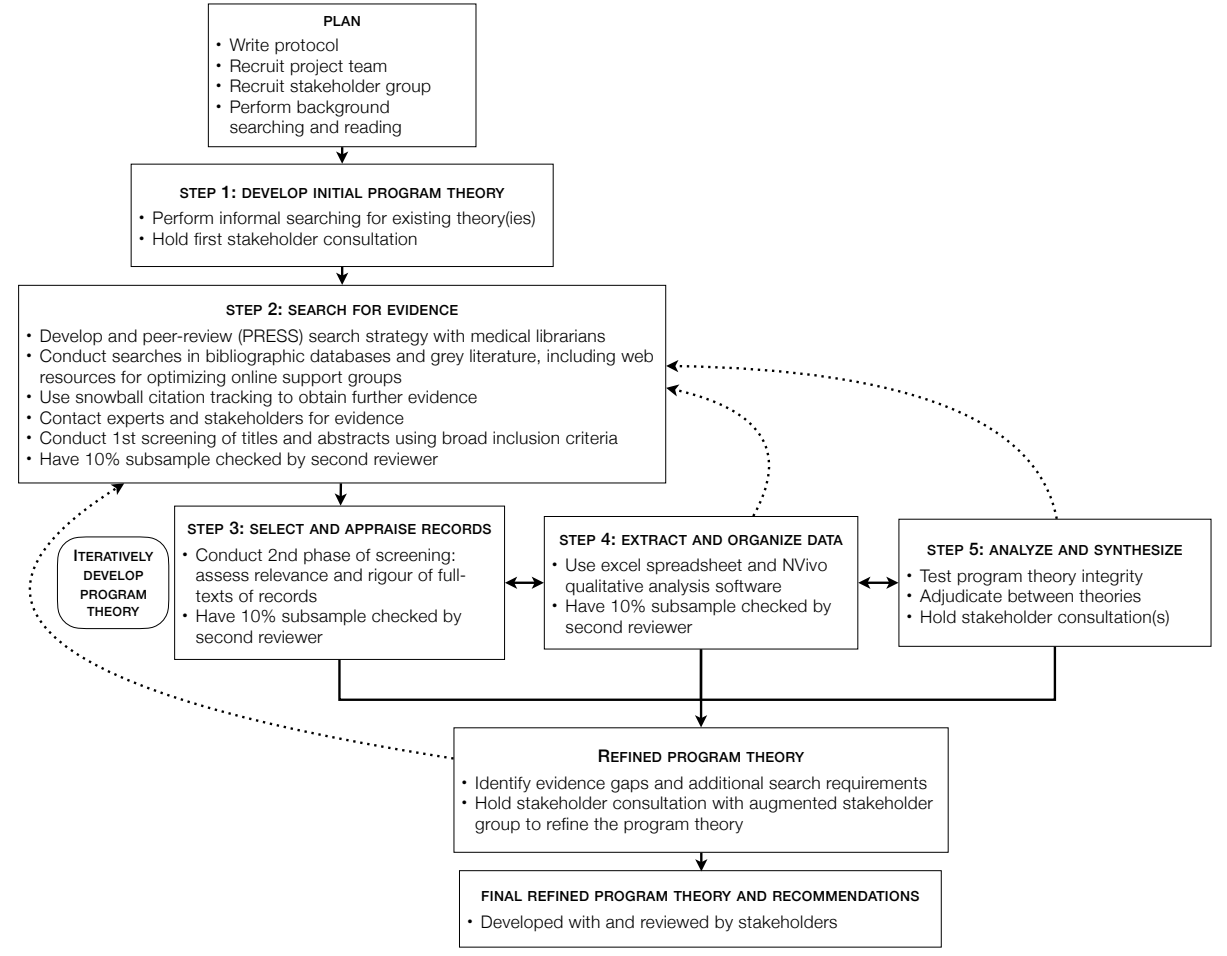

Figure 1 Project overview. Adapted with permission from Duddy and Wong. ${ }^{39}$

that attempts to explain how the intervention works, collecting data from the literature to confirm, refute and refine it, and consulting with stakeholders to ensure the resultant program theory is relevant and resonates with their experiences. ${ }^{36}$ The end product is a middle range theory that it is specific enough to be tested but general enough to be transferable to other settings where the same mechanisms may be in operation. ${ }^{39}$

\section{Objectives}

1. To conduct a realist review of cancer online support groups for people with cancer.

2. To develop a program theory that explains how cancer online support groups work, in what circumstances, for whom and why.

3. To make best practice recommendations based on the findings from the review.

\section{Review questions}

1. What positive and negative outcomes have been reported on cancer online support groups?

2. What are the mechanisms associated with these outcomes, in which contexts, for whom and why?

The review will be conducted according to Pawson $e t$ al's five steps ${ }^{36}$ and will follow the Realist and Meta-narrative Evidence Syntheses Evolving Standards for quality and reporting. ${ }^{40}$ Figure 1 provides a schematic overview of the project.

\section{Stakeholder involvement}

We will adopt an integrated knowledge translation approach, ${ }^{41}$ which is a collaborative approach to research that engages knowledge users as partners in the research process to enhance the relevance and uptake of the findings. This approach aligns with the realist review methodology, which requires ongoing dialogue with stakeholders throughout the project. ${ }^{40}$ Five categories of knowledge users have been engaged in the project: (1) program developers including researchers, clinicians and patient educators from healthcare organisations that develop online support groups (both lay and professionally moderated; standalone and multicomponent interventions); (2) community strategists and moderators that manage and moderate online support groups; (3) clinicians who refer patients to and/or deliver online support groups; (4) patients and family members who have used or created their own 'grassroots' online support groups; and (5) healthcare administrators and policy-makers responsible for implementing online support groups and driving best practices. Representatives from each category of knowledge user, some of whom span multiple categories, are members of the project team or collaborators and will serve as the core stakeholder group.

\section{Patient and public involvement}

We will also follow Canada's Strategy for Patient-Oriented Research Patient Engagement Framework by engaging patients as partners in the research process to ensure the evidence produced focuses on patient-identified priorities and improves patient outcomes. ${ }^{42}$ Three patients with cancer and survivors have been engaged as members of the project team and are part of the core stakeholder group. These individuals include men and women, paediatric, young adult and older adult survivors of lymphoma, breast cancer and prostate cancer, respectively. To 
monitor and improve the patient engagement process, we will track team members' experience of the patientresearcher partnership using patient engagement surveys created by Abelson et al. ${ }^{43}$ Theses anonymous, voluntary surveys will be administered online to all team members at the beginning and at the end of the project.

As shown in figure 1, the core stakeholder group, consisting of knowledge users and patient partners $(\mathrm{n}=15)$, will be consulted multiple times throughout the project when developing the initial program theory, refining the program theory, identifying strategies to disseminate the findings from the review and generating best practice recommendations. In addition, we will hold an augmented stakeholder workshop ${ }^{32}$ towards the end of the study to obtain stakeholder feedback on the program theory and best practice recommendations that we generate. This augmented stakeholder group will include a broader range of stakeholders from our five knowledge user categories with diversity in cancer type, gender and ethnicity. All stakeholder meetings and workshops will be held virtually using web-conferencing software.

\section{Step 1: develop initial program theory}

To develop an initial program theory, ${ }^{38}$ we will: (1) perform an informal scoping search to locate candidate theories and (2) consult stakeholders to assess whether the candidate theory(ies) correspond(s) with their experiences. $^{39}$

To locate existing candidate theories, we will conduct iterative, exploratory searching using structured ${ }^{44}$ and informal search strategies. ${ }^{45}$ We will leverage Bender's multitheory framework on the use and potential effects of breast cancer online support groups ${ }^{24}$ as a starting point. This multitheory framework draws on the Transactional Theory of Stress and Coping, ${ }^{46}$ Social Comparison Theory, ${ }^{47}$ the Technology Acceptance Model $^{48}$ and the Theory of Planned Behaviour. ${ }^{49}$ As shown in figure 2, it suggests that people with breast cancer use online support groups to obtain information and emotional support from similar others to lessen negative appraisals of events, enhance feelings of control, and develop coping strategies, which in turn reduce or buffer anxiety. ${ }^{24}$ The availability, anonymity and low commitment afforded by the online medium are believed to enhance feelings of control. However, the effects of making comparisons with similar others in online support groups may be positive or negative depending on the circumstances and the person's goals. Lastly, beliefs about the usefulness and trustworthiness of online support groups may influence adoption and engagement.

Other potentially valuable theories will include those from the communication and computer sciences, such as Preece's Sociability and Usability framework for determining success in online communities, ${ }^{50}$ as well as the Uses and Gratification Theory for understanding motivations and usage patterns. ${ }^{51}$ In addition, we will consider Perski's theory of engagement in digital behavioural change interventions ${ }^{52}$ to understand how different forms

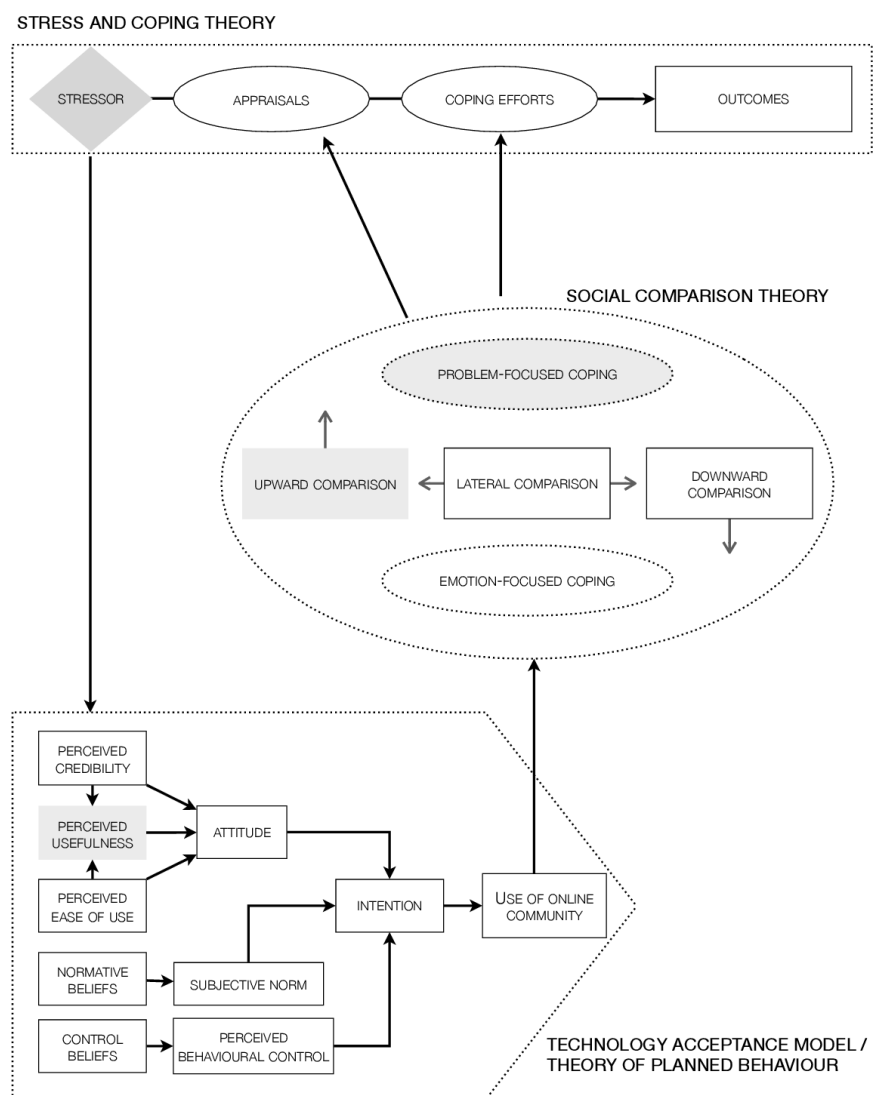

Figure 2 Multitheory framework for understanding the use and effects of online support groups among women with breast cancer. Reproduced with permission from Bender. ${ }^{11} 24$

and degrees of engagement (eg, posting, liking, lurking) may influence outcomes.

We will then hold a stakeholder consultation workshop with the project team and core stakeholder group to gather their views on the processes by which cancer online support groups produce their effects. The workshop will begin with an overview of what is known about cancer online support groups from the literature, followed by break-out groups where team members will discuss their thoughts and experiences. The following questions will guide the break-out group discussions: How do you feel about cancer online support groups? What are some positive outcomes from participating in cancer online support groups? What are some negative outcomes from participating in cancer online support groups? What makes cancer online support groups work well? What makes cancer online support groups work not so well? The break-out groups will be audiorecorded and notes captured using a collaborative note-taking software. The audiorecordings and notes will be analysed using inductive and deductive content analysis. ${ }^{53}$

\section{Step 2: search for evidence}

Following the development of an initial program theory, we will collect evidence to confirm, refute or refine each aspect of theory. ${ }^{35}$ We will conduct a literature search to gather a broad range of heterogeneous data, including quantitative, qualitative and mixed-methods studies, 
peer-reviewed articles, opinion and commentary pieces, as well as grey literature. ${ }^{40}$

\section{Data sources and search strategy}

A search strategy was developed by a medical librarian based on a seminal systematic review of online support groups. ${ }^{28}$ Using this search strategy we conducted a preliminary scoping review of Medline, EMBASE and PsycINFO and found a reasonable number of evaluations of cancer online support groups $(n=61)$. This search strategy was peer-reviewed by an external medical librarian following the Peer Review of Electronic Search Strategy (PRESS) guidelines. ${ }^{54}$ Using this search strategy, searches will be carried out in the following databases from their inception to present: Medline, EMBASE, the Cochrane Database of Systematic Reviews, PsycINFO (all via Ovid), CINAHL (EbscoHost), Scopus (Elsevier) and the Association for Computing Machinery Digital Library. Grey literature will also be retrieved according to the Canadian Agency for Drugs and Technologies in Health's guide. Grey literature will include, for instance, resources on industry websites that offer guidance on building and sustaining online communities (eg, www.feverbee.com).

All abstracts published in English that are captured through database and grey literature searching will be screened according to the inclusion and exclusion criteria outlined below.

\section{Inclusion criteria}

- Study design: Any study design.

- Intervention: An online support group (or an online intervention with an online support group component), defined as a group of individuals with similar or common health-related interests who communicate through a communication platform on the internet.

- Participants: The online support group must be focused on cancer and include people with a cancer diagnosis and/or their family caregivers.

- Outcomes: All types of outcomes associated with participation in online support groups including but not limited to: user engagement, knowledge, selfefficacy, social support, psychological, behavioural or physiological outcomes or use of health services.

\section{Exclusion criteria}

- Studies that report on content analyses of communication occurring between members of an online support group that do not examine the perceived effect of participation in the online support group. For example, content analyses that report on the illness experience, such as the experience of cancer pain, that do not also examine the effect of participating in the online support group on the experience of cancer pain.

\section{Screening process}

Four team members will screen the abstracts using a pilottested screening tool supported by Rayyan web-based software for systematic review management. ${ }^{55}$ Following best practices for abstract screening, ${ }^{56}$ the screening tool will include clear and concise questions organised hierarchically with the easiest questions first. The screening tool will be piloted on five articles prior to screening. To assess inter-rater reliability, all four team members will screen a random sample of 200 articles independently. Inter-rater reliability will be assessed using percent agreement, where greater than $80 \%$ agreement is considered adequate. Once inter-rater reliability is established, the remaining search yield will be divided among the four team members, with the team meeting fortnightly with the project lead to resolve any uncertainties. A $10 \%$ random sample of the total search yield will be audited as is common practice in realist reviews. ${ }^{32}$ Disagreements will be recorded and resolved through discussion.

\section{Additional searching}

More searches will be undertaken if the main search does not generate sufficient data to test the program theory or in response to program theory refinements. ${ }^{57}$ For example, we may need more data to fill a gap in our program theory or investigate a new area of inquiry that our initial search did not find. Where applicable, we will follow cluster search strategies developed by Booth $e t a t^{45}$ to identify and track similar or related sources. Any additional searches will be developed by a medical librarian.

\section{Step 3: selection and appraisal}

Following abstract screening, two team members will read the full texts of all eligible articles, selecting articles for inclusion based on their relevance and rigour. Relevance will be determined by assessing the extent to which the data could contribute to theory building and/or testing by addressing some aspect of the program theory(ies) under consideration. ${ }^{36}$ Rigour will be assessed by determining whether the methods used to produce the relevant data are credible and trustworthy according to the epistemological framework of the study being assessed. ${ }^{36}$ Where necessary, assessments of rigour may involve the use of validated, critical appraisal checklists. ${ }^{57}$ For example, if relevant data have been generated using a questionnaire, then trustworthiness will be considered greater if the questionnaire has been shown to be reliable and valid. A 10\% random subsample will be audited and disagreements will be recorded and resolved through discussion.

\section{Step 4: extracting and organising data}

Following study selection, two team members will extract the main study characteristics (eg, study design (s), participants, intervention, technology, moderation, engagement and outcomes). The extraction tool will be piloted on five articles prior to data extraction. The remaining search yield will be divided among the two team members for independent data extraction, with the pair meeting weekly with the project lead to resolve any uncertainties. As with the previous steps, a $10 \%$ random subsample of extracted data will be audited with disagreements recorded and resolved through discussion. 
One team member will simultaneously upload the included full texts of the articles into NVivo qualitative analysis software to code sections of text that are relevant to supporting, refining or refuting aspects of the program theory. The codes will be conceptual categories that are developed both inductively (codes emerging from the texts) and deductively (codes created in advance and informed by the initial program theory). ${ }^{53}$ This first round of coding will sort data into broad conceptual buckets. Examples of some of the concepts that will be coded are the sex/gender, cancer diagnosis, ethnicity and socioeconomic status of online support group users, type of online support group (eg, standalone/multicomponent, synchronous/asynchronous, lay/professionally moderated) and reasons for using online support groups and outcomes experienced.

\section{Step 5: analysis and synthesis}

In this final step, data that have been sorted into conceptual buckets will be further analysed using a realist logic of analysis. Analysis will be led by a core group of four team members. The goal of this phase of the analysis will be to interpret whether the data are functioning as context, mechanisms or outcomes, and, if so, within which CMOC. ${ }^{36}$ An example CMOC that might be developed is: 'When an online support group moderator welcomes new members (context), the new member starts to open up (outcome) because they feel a sense of psychological safety (mechanism)'. Analysis will involve making judgements about how the CMOCs that are developed fit within the initial program theory. The core stakeholder group will be consulted multiple times during the analysis phase to ensure that the developing program theory fits with their experiences.

\section{Strengths, limitations and mitigation strategies}

A clear scope and plan is critical for the success of this project. ${ }^{365}$ We have built in a number of strategies to ensure its timely completion. Our preliminary scoping review identified a feasible number of articles from the higher yielding sources (eg, Medline EMBASE and PsycINFO). We have identified several promising theories and will use an existing multitheory framework ${ }^{24}$ as a starting point. Further, we have established a core stakeholder group with whom we have worked closely to agree on the focus of the study.

Based on our preliminary scoping review, we expect that most studies of cancer online support groups will focus on women with breast cancer and will include a disproportionate number of white, well educated and above-average income earners. ${ }^{31}$ The predominance of breast cancer-related data may limit the generalisability of findings. However, we anticipate heterogeneity in other respects. This includes variation in the stage of cancer, the communication technology used (eg, discussion form, synchronised chat, microblog community, text/ video, anonymous/semianonymous), the structure and format of the intervention (eg, structured/unstructured, stand-alone/multicomponent, anonymous/semianonymous), the nature and extent of moderation (eg, lay/ professional/none and moderation strategy) and organisational mandate of the online support group. The availability of rich contextual evidence will determine the extent to which we can address all contextual factors.

Lastly, there is a limit to how much ground we can cover in a single realist review. ${ }^{36}$ Hence, we may have to prioritise some aspects of the program theory and set aside others for future research.

\section{ETHICS AND DISSEMINATION}

The outputs of this review will consist of actionable findings in the form of a theoretical framework that explains the important contextual factors and underlying mechanisms of online support groups for cancer patients and survivors. This will underpin an evidence-based 'how-to' guide on ways to improve the development and implementation of cancer online support groups.

In addition, several knowledge products will be produced guided by the knowledge-to-action framework ${ }^{59}$ For each knowledge user group, we will produce tailored knowledge products by adapting the knowledge generated from this review for their specific needs, the context in which it will be used, and assessing barriers to use. This will involve working directly with stakeholders to produce tailored summaries of the findings and identifying and executing dissemination strategies.

For researchers, clinicians and patient educators, two peerreviewed publications will be produced: (1) the study findings from the realist review and the refined program theory and (2) a 'how-to' guide that outlines practical advice to optimise, tailor and implement cancer online support groups. For community strategists/moderators, healthcare administrators, and patients/survivors who run cancer online support groups, we will produce $\mathrm{e}^{3}$ user-friendly summaries of the findings and best practice recommendations.

The results will be communicated through presentations at scientific conferences and publications in open-source journals, newsletters distributed to relevant healthcare professional associations and healthcare organisations, and evidence summaries distributed by partnering healthcare administrators. In addition, social media dissemination and mobilisation strategies will include a tweet chat on the Cancer Survivor Social Media (\#CSSMchat) online community and a webinar hosted by the Mayo Clinic Social Media Network Webinar Series. Knowledge users on the team will be active in identifying and executing additional strategies to reach more audiences.

The use of secondary data for this review precludes the need for ethical approval.

Author affiliations

${ }^{1}$ Cancer Rehabilitation and Survivorship Program, Department of Supportive Care, Princess Margaret Cancer Centre, University Health Network, Toronto, Ontario, Canada 
${ }^{2}$ Dalla Lana School of Public Health, and Institute of Health Policy, Management and Evaluation, University of Toronto, Toronto, Ontario, Canada

${ }^{3}$ Nuffield Department of Primary Care Health Sciences, University of Oxford, Oxford, UK

${ }^{4}$ Li Ka Shing Knowledge Institute, St. Michael's Hospital, Unity Health Toronto, Toronto, Ontario, Canada

${ }^{5}$ Library and Information Services, University Health Network, Toronto, Ontario, Canada

${ }^{6}$ Patient Education and Engagement, University Health Network, Toronto, Ontario,

Canada

${ }^{7}$ Centre for Health Informatics and Multiprofessional Education, University College London, London, UK

${ }^{8}$ Department of Behavioural Science and Health, University College London, London, UK

${ }^{9}$ Department of Psychiatry, University of Toronto, Toronto, Ontario, Canada

${ }^{10}$ Department of Artificial Intelligence and Informatics, Mayo Clinic, Rochester, Minnesota, USA

${ }^{11}$ Department of Cellular and Physiological Sciences, University of British Columbia, Vancouver, British Columbia, Canada

${ }^{12}$ Arthur and Sonia Labatt Brain Tumour Research Centre, The Hospital for Sick Children (SickKids), Toronto, Ontario, Canada

${ }^{13}$ Cancer Education, Princess Margaret Cancer Centre, University Health Network, Toronto, Ontario, Canada

${ }^{14}$ Institute of Health Policy, Management and Evaluation, University of Toronto, Toronto, Ontario, Canada

${ }^{15}$ Patient Education, Ontario Health (Cancer Care Ontario), Toronto, Ontario, Canada

${ }^{16}$ Faculté de médecine de I'Université Laval, Quebec City, Quebec, Canada

Twitter Jacqueline L Bender @benderjack and Colleen Young @colleen_young

Acknowledgements We would like to acknowledge the contribution of Dr Jessie McGowan for her peer review of the MEDLINE search strategy.

Contributors JLB conceived the project and designed the methods for the research with methodological expertise from GW and contributions from ACT, ME, HW, OP, ME, JP, HW. JLB and SB drafted the manuscript, and GW, ACT, ME, ABC, HP, $O P, M J E, C Y, R W, V F, J P, C S, C F, A G-H$ and $H W$ read, revised and approved the final version.

Funding This research is supported by the Canadian Institutes of Health Research Patient-Oriented Research Catalyst Grant Reference \#425 068. ACT holds a Tier 2 Canada Research Chair in Knowledge Synthesis and the Ontario Ministry of Research, Innovation, and Science Early Researcher Award.

Competing interests GW is deputy chair of the UK's National Institute for Health Research's Health Technology Assessment Prioritisation Committee: Integrated Community Health and Social Care (Panel A).

Patient and public involvement Patients and/or the public were involved in the design, or conduct, or reporting, or dissemination plans of this research. Refer to the Methods section for further details.

Patient consent for publication Not applicable.

Provenance and peer review Not commissioned; externally peer reviewed.

Open access This is an open access article distributed in accordance with the Creative Commons Attribution Non Commercial (CC BY-NC 4.0) license, which permits others to distribute, remix, adapt, build upon this work non-commercially, and license their derivative works on different terms, provided the original work is properly cited, appropriate credit is given, any changes made indicated, and the use is non-commercial. See: http://creativecommons.org/licenses/by-nc/4.0/.

\section{ORCID iDs}

Jacqueline L Bender http://orcid.org/0000-0003-1501-335X

Geoff Wong http://orcid.org/0000-0002-5384-4157

Andrea C Tricco http://orcid.org/0000-0002-4114-8971

Marina Englesakis http://orcid.org/0000-0002-2199-1056

Henry Potts http://orcid.org/0000-0002-6200-8804

Olga Perski http://orcid.org/0000-0003-3285-3174

Colleen Young http://orcid.org/0000-0002-9377-7868

Richard Wassersug http://orcid.org/0000-0001-7028-7615

Victoria Forster http://orcid.org/0000-0002-9103-8551

Janet Papadakos http://orcid.org/0000-0001-6320-4156

Charlene Soobiah http://orcid.org/0000-0002-2271-3082
Holly Witteman http://orcid.org/0000-0003-4192-0682

\section{REFERENCES}

1 Bray F, Ferlay J, Soerjomataram I, et al. Global cancer statistics 2018: GLOBOCAN estimates of incidence and mortality worldwide for 36 cancers in 185 countries. CA Cancer J Clin 2018;68:394-424.

2 Global cancer statistics. Lyon, France 2020.

3 Stein KD, Syrjala KL, Andrykowski MA. Physical and psychological long-term and late effects of cancer. Cancer 2008;112:2577-92.

4 Weaver KE, Forsythe LP, Reeve BB, et al. Mental and physical health-related quality of life among U.S. cancer survivors: population estimates from the 2010 National health interview survey. Cancer Epidemiol Biomarkers Prev 2012;21:2108-17.

5 Hewitt M, Greenfield S, Stovall E. From cancer patient to cancer survivor: lost in transition. Washington, D.C.: National Academies Press, 2006.

6 Nekhlyudov L, Ganz PA, Arora NK, et al. Going beyond being lost in transition: a decade of progress in cancer survivorship. J Clin Oncol 2017;35:1978-81.

7 Jefford M, Tattersall MHN. Informing and involving cancer patients in their own care. Lancet Oncol 2002;3:629-37.

8 Shakeel S, Tung J, Rahal R, et al. Evaluation of factors associated with unmet needs in adult cancer survivors in Canada. JAMA Netw Open 2020;3:e200506.

9 Burg MA, Adorno G, Lopez EDS, et al. Current unmet needs of cancer survivors: analysis of open-ended responses to the American cancer Society study of cancer survivors II. Cancer 2015;121:623-30.

10 Bender JL, O'Grady L, Jadad AR. Supporting cancer patients through the continuum of care: a view from the age of social networks and Computer-Mediated communication. Curr Oncol 2008; $15: 107-7$

11 Bender J. Chapter 3: Cancer Virtual Communities in the Era of Personalized Medicine: Perspectives on Scope, Use and Impact. In: El Morr C, ed. Novel applications of virtual communities in healthcare settings, 2018: 56-92.

12 Vivar CG, McQueen A. Informational and emotional needs of longterm survivors of breast cancer. J Adv Nurs 2005;51:520-8.

13 King AJL, Evans M, Moore THM, et al. Prostate cancer and supportive care: a systematic review and qualitative synthesis of men's experiences and unmet needs. Eur $J$ Cancer Care 2015;24:618-34.

14 Arora NK, Finney Rutten LJ, Gustafson DH, et al. Perceived helpfulness and impact of social support provided by family, friends, and health care providers to women newly diagnosed with breast cancer. Psychooncology 2007;16:474-86.

15 Ussher J, Kirsten L, Butow P, et al. What do cancer support groups provide which other supportive relationships do not? The experience of peer support groups for people with cancer. Soc Sci Med 2006;62:2565-76.

16 Berkman LF, Glass T, Brissette I, et al. From social integration to health: Durkheim in the new millennium. Soc Sci Med 2000;51:843-57.

17 Preece J. Online communities: designing usability, supporting sociability. New York: John Wiley \& Sons, Inc, 2000.

18 Sharf BF. Communicating breast cancer on-line: support and empowerment on the Internet. Women Health 1997;26:65-84.

19 Broom A. The eMale. Journal of Sociology 2005;41:87-104.

20 van Uden-Kraan CF, Drossaert CHC, Taal E, et al. Empowering processes and outcomes of participation in online support groups for patients with breast cancer, arthritis, or fibromyalgia. Qual Health Res 2008;18:405-17.

21 Huber J, Muck T, Maatz P, et al. Face-To-Face vs. online peer support groups for prostate cancer: a cross-sectional comparison study. J Cancer Surviv 2018;12:1-9.

22 Kaal SE, Husson O, van Dartel F, et al. Online support community for adolescents and young adults (AYAs) with cancer: user statistics, evaluation, and content analysis. Patient Prefer Adherence 2018;12:2615-22.

23 Vilhauer RP, McClintock MK, Matthews AK. Online support groups for women with metastatic breast cancer: a feasibility pilot study. $J$ Psychosoc Oncol 2010;28:560-86.

24 Bender JL, Katz J, Ferris LE, et al. What is the role of online support from the perspective of facilitators of face-to-face support groups? A multi-method study of the use of breast cancer online communities. Patient Educ Couns 2013;93:472-9.

25 Richardson CR, Buis LR, Janney AW, et al. An online community improves adherence in an internet-mediated walking program. 
Part 1: results of a randomized controlled trial. J Med Internet Res 2010;12:e71.

26 Wootten AC, Meyer D, Abbott J-AM, et al. An online psychological intervention can improve the sexual satisfaction of men following treatment for localized prostate cancer: outcomes of a randomised controlled trial evaluating my road ahead. Psychooncology 2017;26:975-81.

27 Salzer MS, Palmer SC, Kaplan K, et al. A randomized, controlled study of Internet peer-to-peer interactions among women newly diagnosed with breast cancer. Psychooncology 2010;19:441-6.

28 Eysenbach G, Powell J, Englesakis M, et al. Health related virtual communities and electronic support groups: systematic review of the effects of online peer to peer interactions. BMJ 2004;328:1166.

29 Hoey LM, leropoli SC, White VM, et al. Systematic review of peersupport programs for people with cancer. Patient Educ Couns 2008;70:315-37.

30 van Eenbergen MC, van de Poll-Franse LV, Heine P, et al. The impact of participation in online cancer communities on patient reported outcomes: systematic review. JMIR Cancer 2017;3:e15.

31 McCaughan E, Parahoo K, Hueter I, et al. Online support groups for women with breast cancer. Cochrane Database Syst Rev 2017;3:CD011652.

32 Papoutsi C, Mattick K, Pearson M, et al. Interventions to improve antimicrobial prescribing of doctors in training (impact): a realist review. Health Serv Deliv Res 2018;6:1-136.

33 Ford JA, Wong G, Jones AP, et al. Access to primary care for socioeconomically disadvantaged older people in rural areas: a realist review. BMJ Open 2016;6:e010652.

34 Weetman K, Wong G, Scott E, et al. Improving best practice for patients receiving hospital discharge letters: a realist review. BMJ Open 2019;9:e027588.

35 Wong G, Greenhalgh T, Pawson R. Internet-Based medical education: a realist review of what works, for whom and in what circumstances. BMC Med Educ 2010;10:12.

36 Pawson R, Greenhalgh T, Harvey G, et al. Realist review--a new method of systematic review designed for complex policy interventions. J Health Serv Res Policy 2005;10 Suppl 1:21-34.

37 Kastner M, Tricco AC, Soobiah C, et al. What is the most appropriate knowledge synthesis method to conduct a review? protocol for a scoping review. BMC Med Res Methodol 2012;12:114.

38 Pawson R, Tilley N. Realistic evaluation. London, England: Sage Publications, 1997.

39 Duddy $\mathrm{C}$, Wong $\mathrm{G}$. Explaining variations in test ordering in primary care: protocol for a realist review. BMJ Open 2018;8:e023117.

40 Wong G, Greenhalgh T, Westhorp G, et al. RAMESES publication standards: realist syntheses. BMC Med 2013;11:21.

41 Canadian Institutes of Health Research. Guide to knowledge translation planning at CIHR: integrated and End-of-Grant approaches. Ottawa, Canada, 2015.
42 Canadian Institutes of Health Research. Strategy for Patient-Oriented Research (SPOR)- Patient Engagement Framework. Ottawa, Canada, 2014.

43 Abelson J, Li K, Wilson G, et al. Supporting quality public and patient engagement in health system organizations: development and usability testing of the public and patient engagement evaluation tool. Health Expect 2016;19:817-27.

44 Greenhalgh T, Peacock R. Effectiveness and efficiency of search methods in systematic reviews of complex evidence: audit of primary sources. BMJ 2005;331:1064-5.

45 Booth A, Harris J, Croot E, et al. Towards a methodology for cluster searching to provide conceptual and contextual "richness" for systematic reviews of complex interventions: case study (CLUSTER). BMC Med Res Methodol 2013;13:118.

46 Lazarus S. Stress, apprais/ and coping. New York: Springer, 1984

47 Festinger L. A theory of social comparison processes. Human Relations 1954;7:117-40.

48 Davis FD, usefulness P. Perceived usefulness, perceived ease of use, and user acceptance of information technology. MIS Quarterly 1989;13:319-39.

49 Ajzen I. The theory of planned behavior. Organ Behav Hum Decis Process 1991;50:179-211.

50 Preece J. Sociability and usability in online communities: determining and measuring success. Behav Inf Technol 2001;20:347-56.

51 Trowbridge J. The uses of mass communications: current perspectives on Gratifications research. Am J Socio 1976;81:1546-8.

52 Perski O, Blandford A, West R, et al. Conceptualising engagement with digital behaviour change interventions: a systematic review using principles from critical interpretive synthesis. Trans/ Behav Med 2017;7:254-67.

53 Hsieh H-F, Shannon SE. Three approaches to qualitative content analysis. Qual Health Res 2005;15:1277-88.

54 McGowan J, Sampson M, Salzwedel DM, et al. PRESS Peer Review of Electronic Search Strategies: 2015 Guideline Statement. J Clin Epidemiol 2016;75:40-6.

55 Ouzzani M, Hammady H, Fedorowicz Z, et al. Rayyan - a web and mobile APP for systematic reviews. Syst Rev 2016;5:210.

56 Polanin JR, Pigott TD, Espelage DL, et al. Best practice guidelines for Abstract screening large-evidence systematic reviews and metaanalyses. Res Synth Methods 2019;10:330-42.

57 Wong G, Brennan N, Mattick K, et al. Interventions to improve antimicrobial prescribing of doctors in training: the IMPACT (IMProving Antimicrobial presCribing of doctors in Training) realist review: Figure 1. BMJ Open 2015;5:e009059.

58 Grimshaw J. A knowledge synthesis chapter. Ottawa, Canada, 2010.

59 Graham ID, Logan J, Harrison MB, et al. Lost in knowledge translation: time for a MAP? J Contin Educ Health Prof 2006;26:13-24. 